\title{
A Fuzzy Rule Based Expert System for Early Diagnosis of Osgood Schlatter Disease of Knee Joint
}

\author{
Gagandeep Kaur, Lovely Professional University, Phagwara, India \\ Abhinav Hans, Lovely Professional University, Phagwara, India \\ Anshu Vashisth, Lovely Professional University, Phagwara, India
}

\begin{abstract}
The proposed research work is for the early diagnosis of the inflammatory disease named OsgoodSchlatter disease of the knee joint. As the system deals with fuzzy values, a MATLAB (R2013a) fuzzy logic controller is used for the implementation. The knowledge engineering phase is done with the help of an orthopedic expert. Four symptoms are used for diagnosing the severity of disease. Also, this diagnosis provides the treatment for the respective level of disease. Data collection is completed by the survey method and various defuzzification methods are used to check the accuracy. The proposed system was tested on 25 patients.
\end{abstract}

\section{KEYWORDS}

Defuzzification, Fuzzy Logic, Inference Engine, Linguistic Variables, Osgood-Schlatter

\section{INTRODUCTION}

In recent years, many soft computing methods are evolved to improve the quality in medical field. As Medical field is very complex. Nowadays, artificial intelligence (AI) is the one of most emerging fields in all the domains. Expert system which comes under AI, is a human coded program having reasoning capability like human beings (Sethi, 2016). Expert system uses the concept of forward chaining which is a bottom up approach. Expert system is a program that performs the decision-making capabilities by using domain expertise knowledge in form of rules and facts. All the rules provided by domain expert is treated as the knowledge base of the expert system (Khamparia, 2018). From these all collected rules, inference engine which is processing unit of expert system, matches the fact which is stored in working memory. If the rule matches with the fact, then agenda will fire that active rule. The fired rule is basically the consequent part of the rule as the antecedent part is matched with the fact. Expert system provides aids to doctors and users without having knowledge of AI. The proposed system is developed to diagnose the orthopaedic disease named Osgood-Schlatter diseases of the main hinge joint, which is knee joint. Orthopaedic diseases deals with deformities, injuries. All the joint diseases come under the broad category of orthopaedic. The knee joint is comprised of three bones: the femur, tibia, and patella (Pandey, 2009).

\section{DOI: 10.4018/IJHISI.2020040103}

This article, originally published under IGI Global's copyright on December 20, 2019 will proceed with publication as an Open Access article starting on January 14, 2021 in the gold Open Access journal, International Journal of Healthcare Information Systems and Informatics (converted to gold Open Access January 1, 2021), and will be distributed under the terms of the Creative Commons Attribution License (http://creativecommons.org/licenses/by/4.0/) which permits unrestricted use, distribution, and production in any medium, provided the 
Osgood-Schlatter disease is an inflammation disease which occurs mostly in adolescents. Figure 1 shows the affected parts of knee by Osgood-Schlatter disease. This is very common in sports as athletes do vigorous sports activities. This disease is usually more common in boys than girls (Domingues, 2013). It occurs in boys at the age 8-14 and in girls at the age of 10-13. At that age, children are doing many exercises by playing, running, jumping. It causes pain in the lower front part of knee which is tibial tubercle or patellar tendon region. As shown in figure 1 the bone shin inflames.

As a result, the need is for safe and healthy treatments. In artificial intelligence, an expert system is the most emerging field which uses domain expert knowledge to diagnose the disease accurately. In the 1970s when there is beginning of fuzzy logic, the first MES was developed to diagnose the infectious blood disease MYCIN. It was designed using the LISP language and by using 500 rules with various vague and incomplete input values. It deals with certain degrees of the belief using combining functions (Sethi, 2016). With the advancement in AI, many medical expert systems are evolved like DENDRAL, cancer, PROSPECTOR, cardiac disease, ENT, malaria, asthma, dengue, gynecology, and tumors.

In 2002, using fuzzy relational theory author designed the hierarchical fuzzy inference system to diagnose the arthritis disease in various joints. It was two level process, where first level diagnoses reduced the scope of diagnose in second level by taking different parameters (Lim, 2002). In 2016, new intelligent system was developed to diagnose the Inflammatory disease named, Rheumatoid arthritis (RA) of the knee in two stages. In first, image processing technique as RGB of X-rays was used and extracted features were treated as input for neural network (Helwan, 2016). In 2017, author proposed a fuzzy framework in the field of medical imaging to diagnose the Osteoporosis disease, a bone humiliating disease. The silent disease affected regardless of age and gender. This disease was highly found on people over the age of 60 . To calculate the bone density, conventional x-ray image processing technique was used. To analyze trabecular bone texture, resolution enhancement and edge detection algorithm was used. The fuzzy logic controller took 8 input parameters which were considered as risk factors. Testing was conducted on 20 patients which results that the proposed algorithm replaces the existing one (Reshmalakshmi, 2016). The proposed work is designed to diagnose the OSD disease at early stage by using vague values. A rule based expert system is designed to diagnose.

\section{FUZZY SET THEORY}

Fuzzy set theory given by Zadeh makes use of the Vague values (linguistic variables) and degrees of partition to surmount the imprecise, incomplete and uncertain human reasoning in decision making processes. A fuzzy set can be defined as a collection of elements together with their degree of membership. This degree of membership indicates the degree of truth through which a fuzzy element is present in a fuzzy set8. Consider a set A of elements $x$ as defined in Equation (1):

Figure 1. Knee part affected by Osgood Schlatter disease

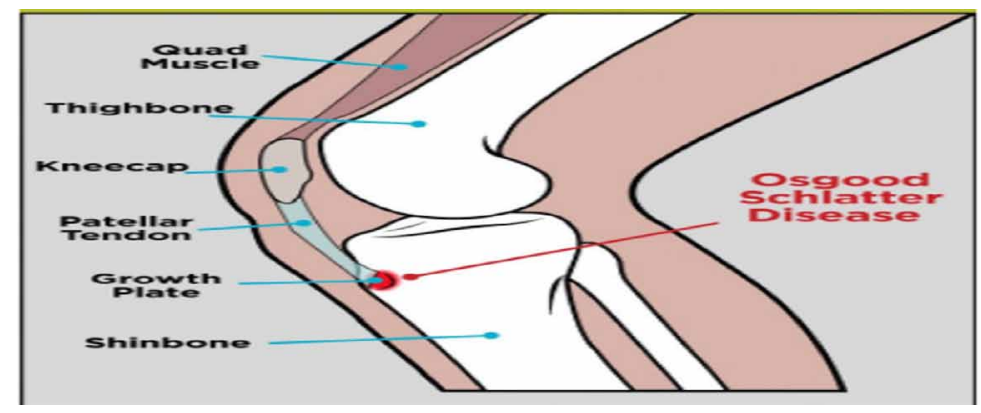




$$
A=\{x \mid x>n\}
$$

A partition function can be defined as given by following Equation (2):

$$
\mu_{K}(x): X \rightarrow[0,1]
$$

Therefore, in set A fuzzy set with elements $x$ membership (partition) value $\mu_{K}(x)$ can be represented by Equation (3):

$$
K=\left\{x, \mu_{K}(x) \mid x \in A\right\}
$$

In 1974, After Zadeh' theory, Mamdani proposed a fuzzy inference process which make use of linguistic control rules. The main parts of Mamdani FIS system are shown in Figure 2.

\section{PROPOSED METHODOLOGY}

The proposed expert system is designed using MATLAB (R2013a) fuzzy toolbox. Fuzzy set which does not have crisp set. Crisp set means having value in yes or no, true or false and good or bad form. It is used where there is confusion either to accept the number or to reject the number which means the number is on boundary. That number which is on the boundary line, it creates confusion whether to accept that or to reject. For these types of input parameters fuzzy set is used. Mamdani inference engine is used for the implementation. Figure 2. shows the methodology of proposed expert system which is designed to diagnose Osgood Schlatter diseases of knee joint.

\section{Knowledge Acquisition}

The data is collected in this phase with the help of expert. It is very important the knowledge engineering has accurate symptoms and types of diseases, so that by using this knowledge expert can make accurate rules. Orthopaedic experts help to give the adequate information about disease. The expert defines the primary symptoms, range and level of disease (Sethi, 2016). Figure 3 shows knee

Figure 2. Proposed methodology

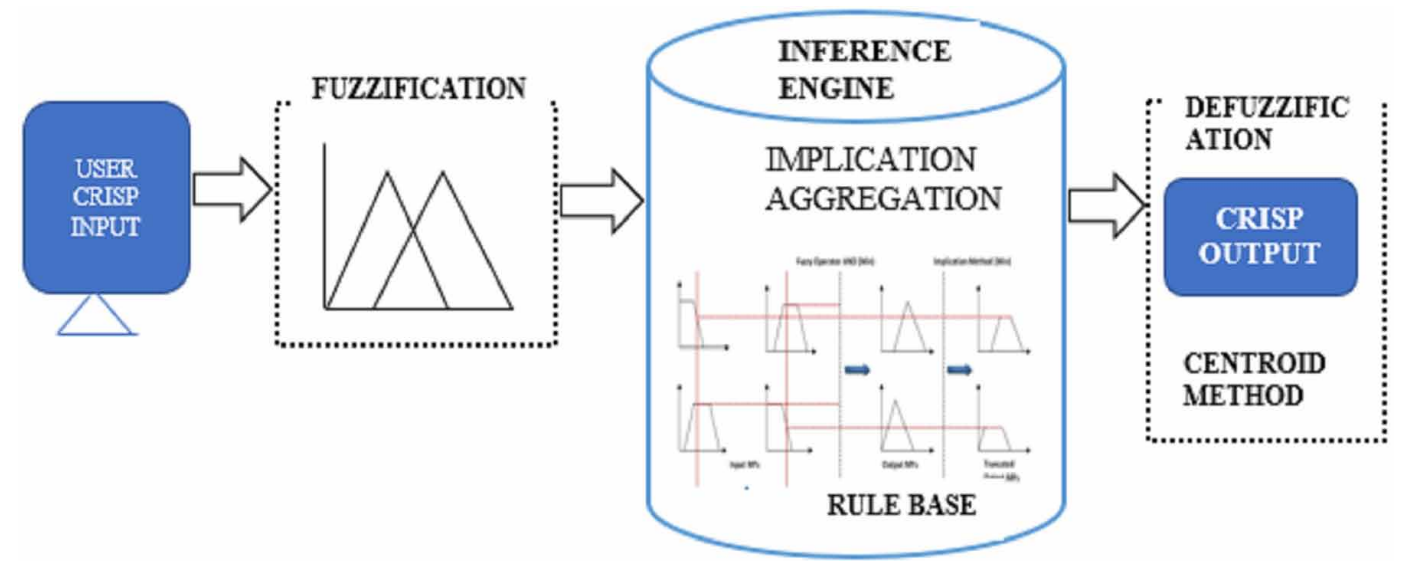




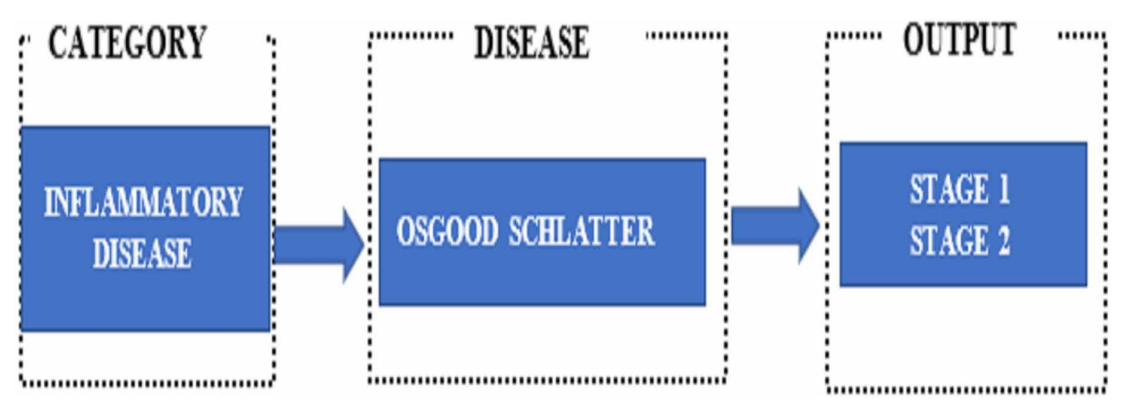

category under which that disease lies, and corresponding output of the disease is shown. Table 1. shows the primary symptoms collected during knowledge acquisition phase.

\section{Fuzzification of Inputs and Outputs}

Fuzzification is second step, where the crisp input is changed to fuzzified value using partition function under degree of belongingness. To handle the value values, there are many membership functions available in Matlab toolbox such as triangular, trapezoidal, gaussian, z-shape and sigmoidal. In this proposed system, triangular and trapezoidal membership functions are used to set range for input and output membership functions. For the input parameter pain, three membership functions are used as shown in Figure 4. For the output variable two membership functions are used as describes in Figure 5.

A trapezoidal membership function can be defined as Equation (1) (Kaur, 2016):

$\mu(x: i, j, k, l)=\max \left(\min \left(\frac{x-i}{j-i}, 1 \frac{l-x}{l-k}\right), 0\right)$

The triangular membership function can be defined as Equation (2) (Kaur, 2016):

$\mu(x: i, j, k)=\max \left(\min \left(\frac{x-i}{j-i}, \frac{k-x}{k-j}\right), 0\right)$

For all the input and output parameters range is set. This range define that these parameters are vague values or linguistic variables. Table 2 shows fuzzified input symptoms and output level for Osgood Schlatter disease of the knee joint.

For the Figure 4, following Equation (6) shows values for first membership function, Equation (8) for second function and Equation (7) for third membership function:

Table 1. Refinement of symptoms

\begin{tabular}{|c|c|}
\hline Disease & Symptoms \\
\hline Osgood-Schlatter & $\begin{array}{l}\text { - Knee Pain } \\
\text { - Tenderness } \\
\text { - Swelling } \\
\text { - Muscle Contraction }\end{array}$ \\
\hline
\end{tabular}


Figure 4. Membership function for input parameter

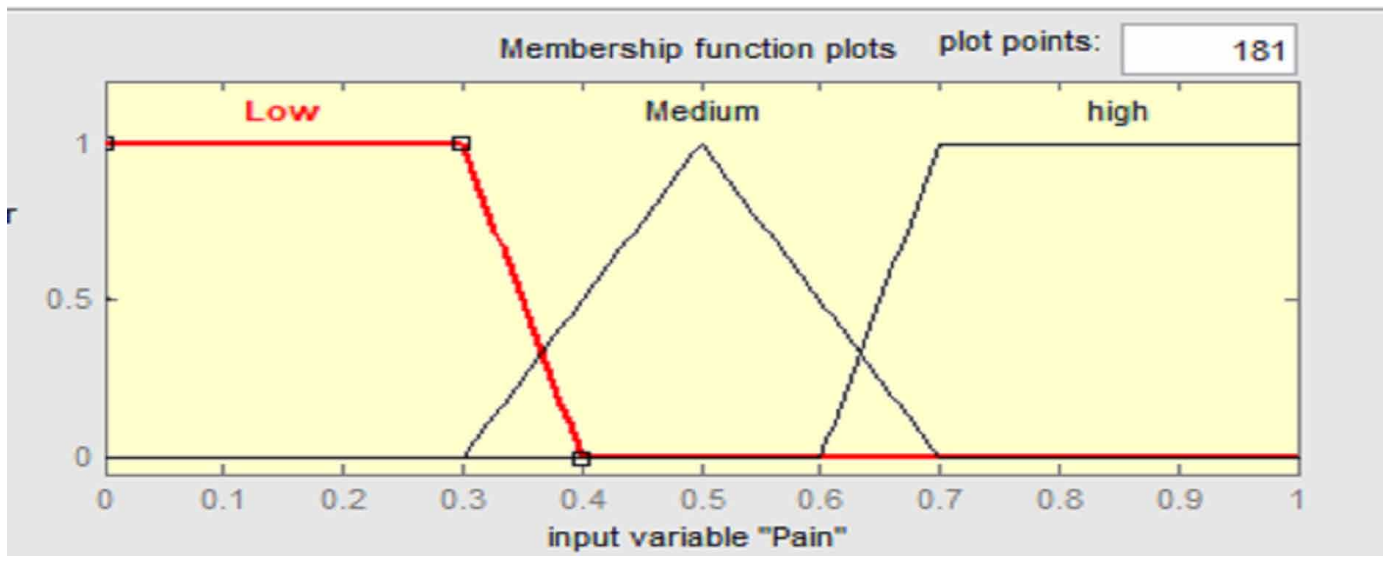

Figure 5. Membership function for output parameter

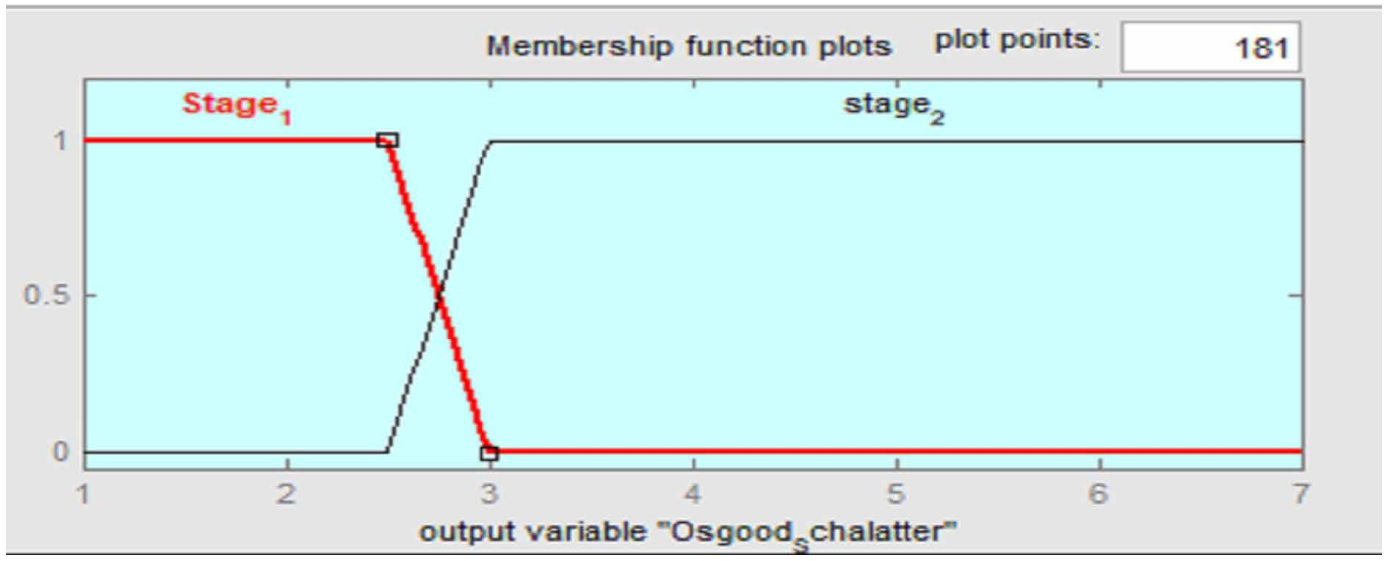

Table 2. Fuzzified input symptoms and output for Osgood Schlatter Disease

\begin{tabular}{|c|c|c|c|c|}
\hline \multicolumn{4}{|c|}{ Inputs } & \multirow{2}{*}{$\frac{\text { Output }}{\text { Osgood Schlatter }}$} \\
\hline Pain & Tenderness & Swelling & Muscle Contraction & \\
\hline $\begin{array}{l}\text { Low } \\
(0-0.4)\end{array}$ & $\begin{array}{l}\text { Low } \\
(0-0.4)\end{array}$ & $\begin{array}{l}\text { Low } \\
(0-0.4)\end{array}$ & $\begin{array}{l}\text { Low } \\
(0-0.4)\end{array}$ & $\begin{array}{l}\text { STAGE } 1 \\
(1-3)\end{array}$ \\
\hline $\begin{array}{l}\text { Medium } \\
(0.3-0.7)\end{array}$ & $\begin{array}{l}\text { Medium } \\
(0.3-0.7)\end{array}$ & $\begin{array}{l}\text { Medium } \\
(0.3-0.7)\end{array}$ & $\begin{array}{l}\text { Medium } \\
(0.3-0.7)\end{array}$ & \multirow{2}{*}{$\begin{array}{l}\text { STAGE } 2 \\
(2.5-7)\end{array}$} \\
\hline $\begin{array}{l}\text { High } \\
(0.6-1)\end{array}$ & $\begin{array}{l}\text { High } \\
(0.6-1)\end{array}$ & $\begin{array}{l}\text { High } \\
(0.6-1)\end{array}$ & $\begin{array}{l}\text { High } \\
(0.6-1)\end{array}$ & \\
\hline
\end{tabular}




$$
\begin{gathered}
\mu_{\text {LOW }}(x)= \begin{cases}\frac{0.4-x}{0.1}, & 0.3 \leq x \leq 0.4 \\
1, & x \leq 3\end{cases} \\
\mu_{\text {HIGH }}(x)= \begin{cases}\frac{x-0.6}{0.1}, & 0.6 \leq x \leq 0.7 \\
1, & x \geq 0.7\end{cases} \\
\mu_{\text {MEDIUM }}(X)= \begin{cases}\frac{x-0.3}{0.4-0.3}, & 0.3 \leq x \leq 0.4 \\
\frac{0.7-x}{0.7-0.6}, & 0.6 \leq x \leq 0.7\end{cases}
\end{gathered}
$$

\section{Fuzzy Rule Base Model}

After fuzzification, next step is making Knowledge base. It is formed from the fuzzy rules (Walia,2015). Expert system makes the decisions or reasoning based on these fuzzy rules. Fuzzy rules for the proposed medical expert system are constructed by consulting the Orthopaedic expert. With the combination of all input parameters, total 81 rules are made to diagnose the stage of disease. Some sample rules are shown below:

- If (Pain is high) AND (Tenderness is high) AND (Swelling is Low) AND (Muscle Contraction is Medium) THEN (Osgood Schlatter is STAGE 2);

- If (Pain is high) AND (Tenderness is high) AND (Swelling is Low) AND (Muscle Contraction is Low) THEN (Osgood Schlatter is STAGE 1);

- If (Pain is Medium) AND (Tenderness is Low) AND (Swelling is high) AND (Muscle Contraction is Low) THEN (Osgood Schlatter is STAGE 1).

Figure 6 shows the fuzzy rules, Figure 7 shows the rule viewer and Figure 8 shows surface viewer of proposed expert system.

\section{Figure 6. Fuzzy rules}

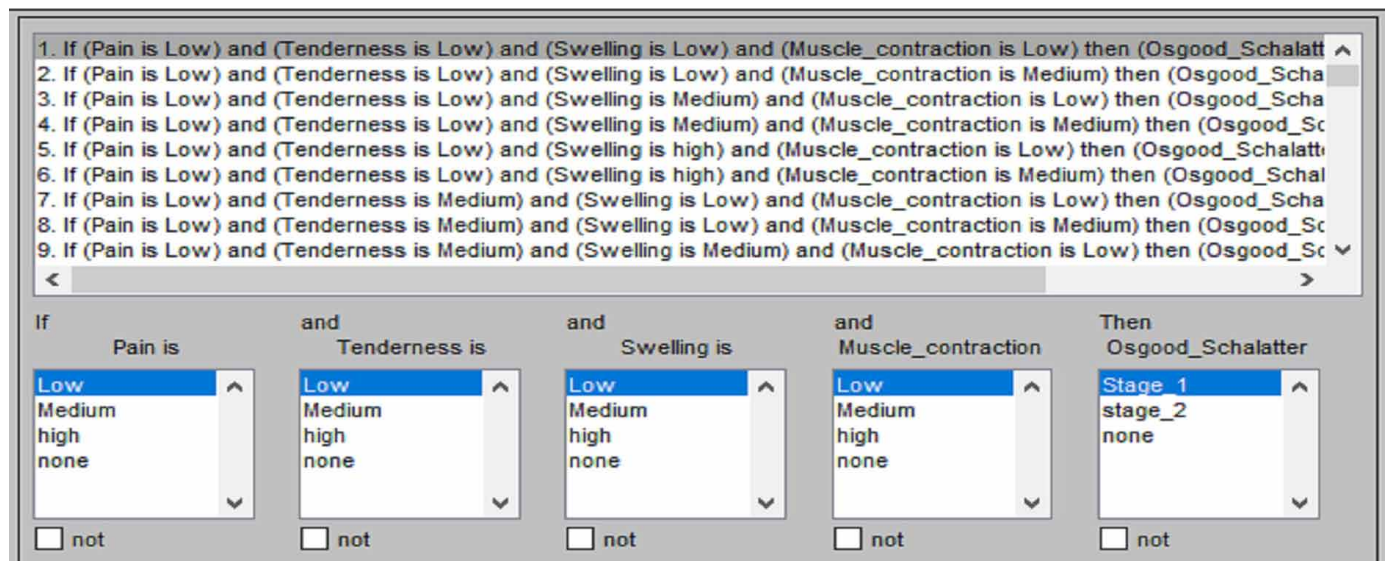


Figure 7. Rule viewer

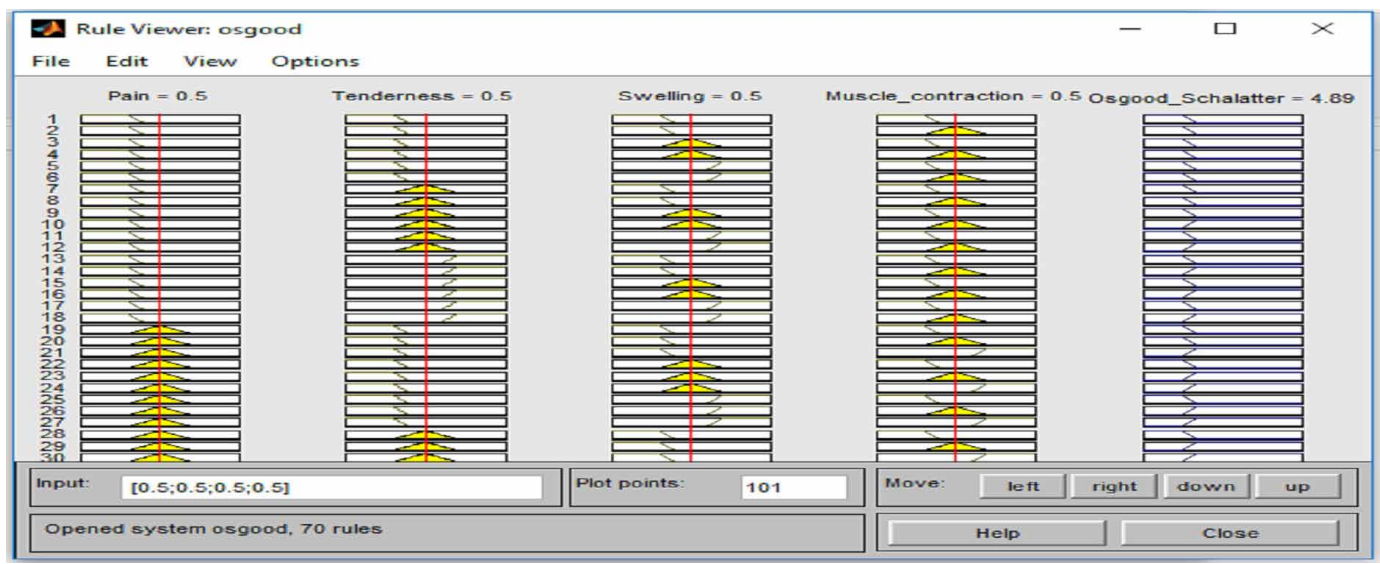

Figure 8. Surface viewer

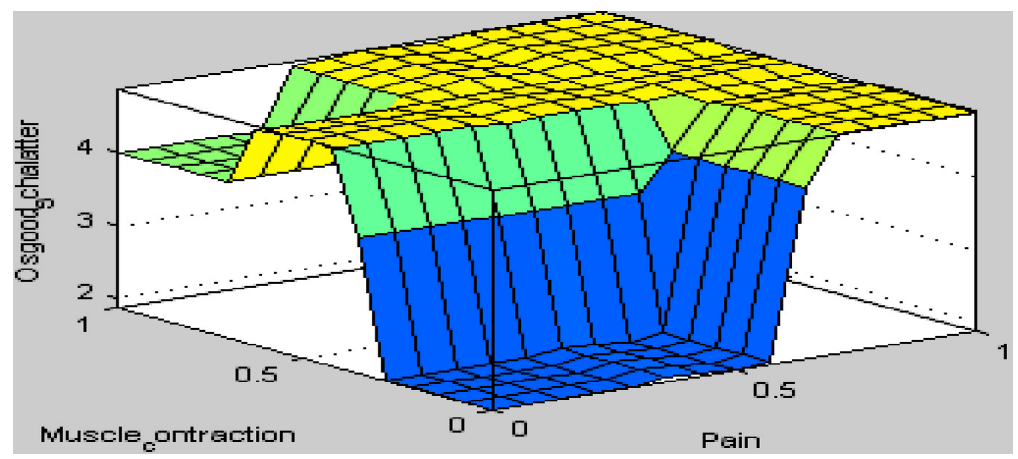

\section{Fuzzy Inference}

Mamdani Fuzzy Inference system is used for the fuzzy Methodology as it deals with fuzzy logic or linguistic variables, which is represented by following Equation (9) (Kaur, 2016):

$$
f_{C}(z)=\frac{\max }{k}\left[\min \left[f_{A}(\operatorname{input}(i)), f_{B}(\text { input }(j))\right]\right], k=1,2,3 \ldots \ldots n
$$

In fuzzy inference process, basically two methods are used: Implication Method and Aggregation Method. The fuzzy rules make using linguistic variables are combined with fuzzy operators (AND, OR) [9]. In the implication method, if AND operator is used, it will return the Minimum value for that variable and for OR operator maximum value is obtained. As a result, if more than one rule is matched the single output is generated. The process of prioritize the rules are done by Agenda. It could be done based on the value that is set for each rule. The rule having higher priority will be chosen as output. The implication process is evaluated for each rule to generate output membership function. After the implication, next step is aggregation, which receive all the truncated output produced by implication method and output as a fuzzy set. Figure 9 shows the fuzzy inference for proposed system which takes 4 inputs and one output variable. 


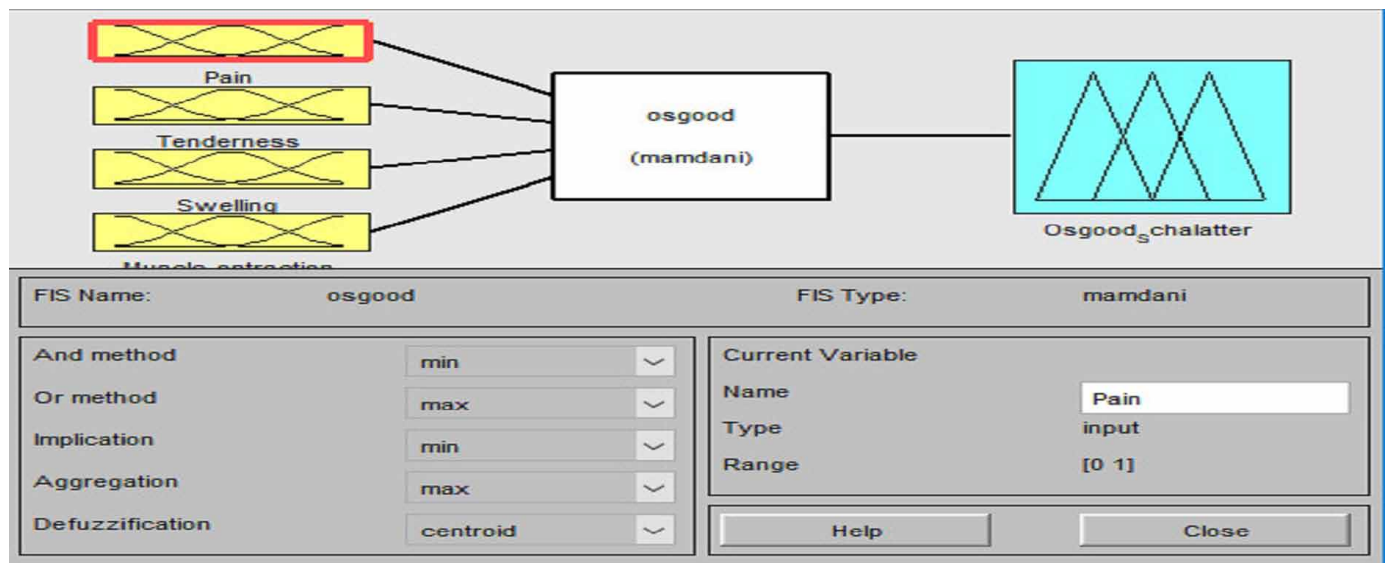

\section{Defuzzification}

The last step is defuzzification, which is used to get the crisp output from the fuzzified value (Madaan,2016). It is necessary step because the user can understand crisp value. Matlab provides five defuzzification methods as defined below:

- Center of area (COA): Centroid method also called 'center of gravity' as it calculates the centroid for that area which is under aggregation output membership function:

$$
Z_{C O A}=\frac{\int \mu_{A}(z) z d z}{\int \mu_{A}(z) z d z}
$$

- Bisector of area (BOA): Equal part of whole region are made, sometimes the center of area and bisector of area lies on one line but not always:

$$
\int_{a}^{B O A} \mu_{A} d z=\int_{B O A}^{\beta} \mu_{A}(z) d z
$$

- Largest of Maximum (LOM): Largest value is return. This method doesn't require any calculations;

- Smallest of Maximum (SOM): This method of defuzzification doesn't require any calculation as like largest of maximum methods and returns the smallest value;

- Mean of Maximum (MOM): This function returns the value by calculating all maximum values, arithmetic mean of the aggregated membership functions:

$$
Z_{m o m}=\frac{\int z d z}{\int d z}
$$

Table 3 shows Defuzzified values for Osgood Schlatter disease by comparing with all the five defuzzification methods which are discussed. 
Table 3. Defuzzified values for Osgood Schlatter Disease

\begin{tabular}{|l|l|l|l|l|l|l|l|l|l|l|l|l|l|l|l|l|}
\hline \multicolumn{9}{|c|}{ Inputs Symptoms } & \multicolumn{7}{c|}{ Defuzzified Values According to Different Defuzzification } \\
Methods
\end{tabular}

\section{INTERFACE}

The interaction between user and expert system is provided by the help of Graphical user interface (GUI) which refers to series of forms (Amiri,2017). It should be very simple to interact for the users. GUI is a way through which the user can enter the input corresponding to all input variables which are symptoms and the expert system will evaluate and generate the output of the disease. The treatment plan is also shown to the user as shown in Figure 11.

\section{TESTING}

In the medical field, there should be accurate results as it deals with human's life. So, the proposed system is tested with the expert's output. The accuracy is shown as False positive and False negative in Table 4. Table 5 shows the sample results for fuzzy inference system and Table 6 shows patient data for fuzzy inference system:

SENSITIVITY $=$ True Positive $/($ True Positive + False Negative $)=19 /(19+1)=0.95$ SPECIFICITY $=$ True Negative $/($ True Negative + False Positive $)=4 /(4+1)=0.8$

Figure 10. Graphical user interface for Osgood Schlatter Disease

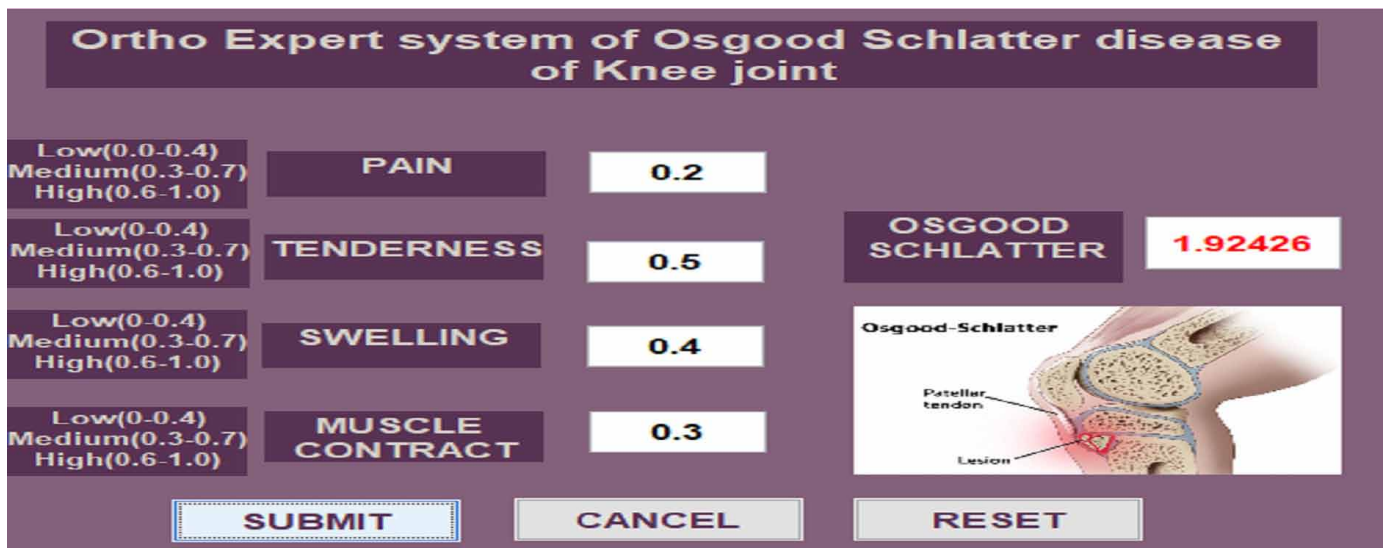


Figure 11. Treatment plan for Osgood Schlatter Disease

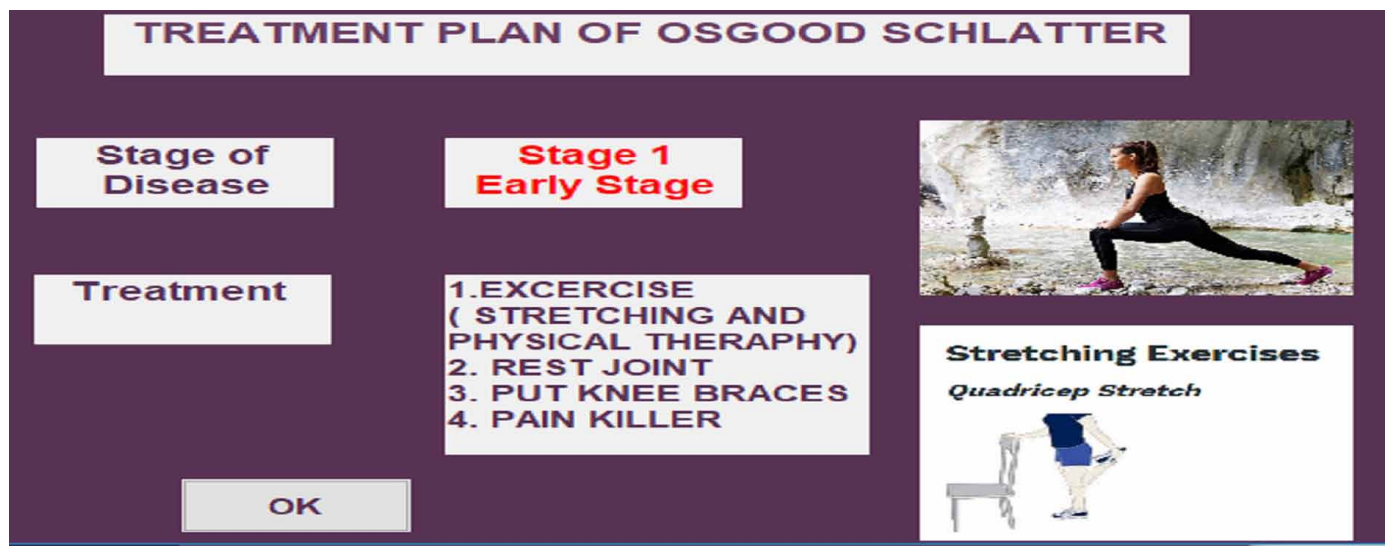

Table 4. Sample test results

\begin{tabular}{|l|l|l|}
\hline \multicolumn{1}{|c|}{ Test } & \multicolumn{1}{|c|}{ Disease Present } & \multicolumn{1}{c|}{ Disease Absent } \\
\hline Test Positive & True Positives (19) & False Negatives (1) \\
\hline Test Negative & False Positives (1) & True Negatives (4) \\
\hline Total & 20 & 5 \\
\hline
\end{tabular}

Table 5. Sample test results for Fuzzy inference system

\begin{tabular}{|l|l|l|l|l|l|l|}
\hline \multicolumn{4}{|c|}{ Inputs Symptoms } & \multicolumn{3}{c|}{ Output } \\
\hline Pain & Tenderness & Swelling & $\begin{array}{c}\text { Muscle } \\
\text { Contraction }\end{array}$ & $\begin{array}{c}\text { Output by } \\
\text { System }\end{array}$ & $\begin{array}{c}\text { Output by } \\
\text { Expert }\end{array}$ & Result \\
\hline 0.5 & 0.2 & 0.7 & 0.9 & 4.88 & Stage 2 & TRUE \\
\hline 0.2 & 0.4 & 0.5 & 0.1 & 1.92 & Stage 1 & TRUE \\
\hline 0.6 & 0.5 & 0.4 & 0.85 & 4.82 & Stage 2 & TRUE \\
\hline 0.2 & 0.7 & 0.8 & 0.9 & 4 & Stage 2 & TRUE \\
\hline 0.7 & 0.1 & 0.2 & 0.5 & 1.86 & Stage 1 & TRUE \\
\hline
\end{tabular}

Table 6. Patient data for Fuzzy inference system

\begin{tabular}{|l|l|l|l|l|l|l|}
\hline \multicolumn{4}{|c|}{ Inputs Symptoms } & \multicolumn{1}{c|}{ Output } \\
\hline Pain & Tenderness & Swelling & $\begin{array}{c}\text { Muscle } \\
\text { Contraction }\end{array}$ & $\begin{array}{c}\text { Output by } \\
\text { System }\end{array}$ & $\begin{array}{c}\text { Output by } \\
\text { Expert }\end{array}$ & Result \\
\hline High & High & High & Medium & Stage 2 & Stage 2 & TRUE \\
\hline High & Low & Low & Low & Stage 1 & Stage 1 & TRUE \\
\hline Low & Low & Medium & Low & Stage 1 & Stage 1 & TRUE \\
\hline Low & High & Medium & Medium & Stage 1 & Stage 2 & TRUE \\
\hline Medium & Medium & Low & Low & Stage 1 & Stage 1 & TRUE \\
\hline
\end{tabular}




\section{DISCUSSION AND CONCLUSION}

The proposed expert system is designed to diagnose the orthopaedic disease of the knee joint which comes under inflammatory category named as Osgood Schlatter disease. It is common in children usually in boys as boys plays more sports than girls. Fuzzy rule base approach is used as it gives accurate results by calculating sensitivity and specificity. Also, it is easy to implement. The system describes the stage of disease based on the knowledge given by expert.

The proposed system is developed to facilitate the orthopaedic experts to determine the stage of OSD disease by entering the symptoms on interface. Therefore, this proposed system could be used as a tool which can assist orthopaedic doctors as well as a learning and educative system for medical students. Also, a layman can use the system to diagnose the disease and its treatment without having any knowledge of artificial intelligence.

The proposed can be further extended by taking more input parameters which are used for evaluating the disease. The system can also be implemented by neuro fuzzy technique and can be tested on a greater number of patients to get more accurate results.

\section{ACKNOWLEDGMENT}

I would like to express my deep sense of gratitude towards Dr. Surinder Singh Dardhi, Orthopaedic Medical Officer, at Sant Sarwan Dass Charitable Hospital, Kathar, Hoshiarpur, Punjab, India who will be providing their expertise throughout the research process. 


\section{REFERENCES}

Amiri, F. M., \& Khadivar, A. (2017). A fuzzy expert system for diagnosis and treatment of musculoskeletal disorders in wrist/Fuzzy ekspertni sustav za dijagnozu i lijecenje misicnoskeletnih poremecaja u rucnom zglobu. Tehnicki Vjesnik-Technical Gazette, 24(S1), A147-A147. https://www.braceability.com/knee-injuries-problems/ osgood-schlatter-disease

Domingues, M. (2013). Osgood Schlatter's disease -A burst in young football players. Journal of Sports Science \& Medicine, 2(1), 23-27.

Helwan, A., \& Tantua, D. P. (2016). IKRAI: Intelligent knee rheumatoid arthritis identification. International Journal of Intelligent Systems and Applications, 8(1), 18.

Kaur, R., Madaan, V., \& Agrawal, P. (2016). Fuzzy expert system to calculate the strength/ immunity of a human body. Indian Journal of Science and Technology, 9(44). doi:10.17485/ijst/2016/v9i44/105145

Khamparia, A., \& Pandey, B. (2017). Effects of visual mapping placed game-based learning on students learning performance in defence-based courses. Int. J. Technology Enhanced Learning, 9(1), 37-50. doi:10.1504/ IJTEL.2017.084083

Khamparia, A., \& Pandey, B. (2018). SVM and PCA Based Learning Feature Classification Approaches for E-Learning System. International Journal of Web-Based Learning and Teaching Technologies, 13(2), 32-45. doi:10.4018/IJWLTT.2018040103

Lim, C. K., Yew, K. M., Ng, K. H., \& Abdullah, B. J. J. (2002). A proposed hierarchical fuzzy inference system for the diagnosis of arthritic diseases. Australasian Physical \& Engineering Sciences in Medicine, 25(3), $144-150$. doi:10.1007/BF03178776 PMID:12416592

Pandey, S., \& Pandey, A. (2009). Clinical orthopaedic diagnosis. New Delhi: Jaypee Brothers Medical Publishers. doi:10.5005/jp/books/11786

Ranjit, K., Vishu, M., Prateek, A., Sanjay Kumar, S., \& Amandeep, K. (2016). Fuzzy Expert System for Identifying the Physical Constituents of a Human Body. Indian Journal of Science and Technology, 9(28). doi:10.17485/ ijst/2016/v9i28/98390

Reshmalakshmi, C., \& Sasikumar, M. (2016). Fuzzy inference system for osteoporosis detection. In Proceedings of GHTC 2016 - IEEE Glob. Humanit. Technol. Conf. Technol. Benefit Humanit. Conf. Proc. (pp. 675-681).

Sethi, D., Agrawal, P., \& Madaan, V. (2016). X-Tumour. Fuzzy Rule Based Medical Expert System To Detect Tumours In, 9(11), 5073-5084.

Sethi, D., Agrawal, P., Madaan, V., \& Kumar Singh, S. (2016). X-Gyno: Fuzzy Method based Medical Expert System for Gynaecology. Indian Journal of Science and Technology, 9(28). doi:10.17485/ijst/2016/v9i28/98387

Singh, A., \& Pandey, B. (2014). Intelligent techniques and applications in liver disorders: A survey. International Journal of Biomedical Engineering and Technology, 16(1), 27-70. doi:10.1504/IJBET.2014.065638

Takkar, S., Singh, A., \& Pandey, B. (2017). Application of machine learning algorithms to a well defined clinical problem: Liver disease. International Journal of E-Health and Medical Communications, 8(4), 38-60. doi:10.4018/IJEHMC.2017100103

Walia, N., Tiwari, S. K., \& Malhotra, R. (2015). Design and Identification of Tuberculosis using Fuzzy Based Decision Support System. Adv. Comput. Sci. Inf. Technol., 2(8), 57-62. 